\title{
Multigene phylogenies for the earthworm Eisenia nordenskioldi (Lumbricidae, Annelida)
}

\author{
Sergei V. Shekhovtsov \\ Kurchatov Genomics Center, \\ ICG SB RAS, \\ Novosibirsk, Russia \\ shekhovtsov@bionet.nsc.ru
}

\author{
Alexandra A. Shipova \\ Kurchatov Genomics Center, ICG SB RAS, \\ Novosibirsk, Russia \\ shipova@bionet.nsc.ru \\ Sergei E. Peltek \\ Kurchatov Genomics Center, \\ ICG SB RAS, Novosibirsk, Russia \\ peltek@bionet.nsc.ru
}

Tatiana V. Poluboyarova

ICG SB RAS, Novosibirsk, Russia poluboyarova@bionet.nsc.ru

\begin{abstract}
Eisenia nordenskioldi is a polymorphic earthworm species with two subspecies and high cryptic genetic diversity. We performed transcriptome sequencing for eleven genetic lineages of this species and built phylogenies based on different extracted datasets. We demonstrated that, contrary to previous studies, $E$. nordenskioldi is monophyletic, but both of its subspecies are polyphyletic. $E$. nordenskioldi was split into two groups of lineages, representing the northwestern and southeastern parts of its range.
\end{abstract}

Keywords - earthworms, Eisenia nordenskioldi, phylogeny, transcriptomes

Motivation and aim

\section{Motivation}

Eisenia nordenskioldi was long known to be a polymorphic species with multiple morphological forms and chromosomal races. A series of recent studies uncovered high cryptic DNA diversity within this species [1-2]. However, phylogenetic inferences using conventional approaches were inconclusive.

Aim

Studies that employed a few genes or mitochondrial genomes failed to resolve the relationships among genetic lineages and subspecies constituting E. nordenskioldi [2-3]. Therefore, we performed transcriptome sequencing and extracted multigene datasets in order to elucidate phylogenetic relations this species.

\section{Methods}

Total RNA was extracted from live specimens; the poly-A RNA was isolated using commercial kits and sequenced by IonTorrent (four specimens) and Illumina HiSeq (seven specimens). The obtained reads were assembled by Trinity.
Gene and protein datasets were extracted using the ProteinOrtho and HaMStR programs for ortholog assignment. Phylogenetic trees were built using Bayesian inference and Maximum Likelihood algorithms.

\section{Results}

Within the studied dataset $E$. nordenskioldi was monophylic, albeit not with high statistical support. E. n. nordenskioldi was paraphyletic with respect to E. n. pallida. Lineages constituting the latter subspecies did not for a monophyletic group. This suggests that the ancestor of E. nordenskioldi was pigmented, and pigmentation was independently lost during the evolution of the species in several distinct lineages. E. n. pallida is thus an artificial group. E. nordenskioldi was split into two groups of lineages, representing the northwestern and southeastern parts of its range.

\section{Acknowledgment}

Supported by the RFBR (19-54-04006_Bel_mol_a, 19-0400661_a) and by Budget Project of FIZ IC $\overline{C i G ~ S B ~ R A ̄ S ~(0324-~}$ 2019-0040-C-01).

\section{References}

[1] Shekhovtsov S.V. et al. (2013) Cryptic diversity within the Nordenskiold's earthworm, Eisenia nordenskioldi subsp. nordenskioldi (Lumbricidae, Annelida). European Journal of Soil Biology. 58:13-18.

[2] Shekhovtsov S.V. et al. (2016) Cryptic genetic lineages in Eisenia nordenskioldi pallida (Oligochaeta, Lumbricidae). European Journal of Soil Biology. 75:151-156.

[3] Shekhovtsov S.V. et al. (2020) Phylogeny of the Eisenia nordenskioldi complex based on mitochondrial genomes. European Journal of Soil Biology. 96:103137. 